\title{
Complete Occlusion of Aortic Arch Branch Vessels Diagnosed by Intraoperative Point-of-Care Ultrasonography: A Case Report
}

\author{
Masafumi Idei $^{1 *}$, Rie Yoshida ${ }^{1}$, Tasuku Yoshida ${ }^{2}$, Itaru Watanabe ${ }^{1}$

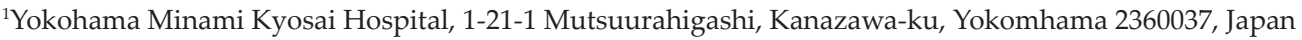 \\ ${ }^{2}$ Yokohama City University Hospital, 3-9 Fukuura, Kanazawa-ku, Yokohama 236-0004, Japan \\ ${ }^{*}$ Corresponding author: Masafumi Idei, Yokohama Minami Kyosai Hospital, 1-21-1 Mutsuurahigashi, Kanazawa-ku, Yokomhama 2360037, Japan; \\ E-mail: masafumi203@gmail.com
}

Received: December 31, 2018; Accepted: January 09, 2019; Published: January 29, 2019;

\begin{abstract}
The use of point-of-care ultrasonography (POCUS) is becoming increasingly widespread and clinically important. Here, we report a case in which the use of POCUS in the operating room identified a previously undiagnosed bilateral subclavian artery stenosis. A 70-year-old woman with a diagnosis of meningioma was scheduled to undergo a craniotomy. After the induction of general anesthesia, an abnormal radial artery waveform was observed bilaterally. A blood flow deficiency in both upper extremities was suspected, and POCUS was immediately performed. Using POCUS, the anesthesiologist identified stenosis and hypoplasia of the bilateral subclavian arteries, complete occlusion of the bilateral common carotid arteries, and remarkably dilated bilateral vertebral arteries. By identifying these vascular abnormalities with POCUS, the anesthesiologist was able to adjust the patient's perioperative circulatory management and lead to a positive postoperative outcome.
\end{abstract}

\section{Background}

Point-of-care ultrasonography (POCUS) is becoming progressively more widespread and clinically valuable [1]. The use of POCUS by anesthesiologists and critical care physicians may help improve outcomes in surgical and critically ill patients [2,3]. During the perioperative period, POCUS is a very efficient and beneficial tool that can be used to diagnose previously unrecognized clinical conditions. However, to the best of our knowledge, there are very few reports in which previously undiagnosed vascular abnormalities of aortic arch branch vessels were identified by POCUS performed by anesthesiologists in the operating room. Here, we report a case in which we suspected bilateral subclavian artery stenosis based on abnormal bilateral radial artery pressure waveforms after induction of anesthesia, immediately performed POCUS, and identified a previously undiagnosed bilateral subclavian artery stenosis.

\section{Case presentation}

A 70-year-old woman diagnosed with a meningioma was scheduled to undergo a craniotomy. In addition to the meningioma, preoperative head magnetic resonance imaging (MRI) revealed that bilateral common carotid arteries were abnormally narrow and bilateral vertebral arteries were abnormally dilated; the blood vessels below the neck were not evaluated. She was alert and oriented with no neurological abnormalities, and the results of the Allen's test were negative.

Her noninvasive blood pressure, measured from the left upper arm at admission, was $139 / 111 \mathrm{~mm} \mathrm{Hg}$. After induction of general anesthesia, a 20 -gauge catheter was inserted in her left radial artery, and the arterial blood pressure was 79/54 $\mathrm{mm} \mathrm{Hg}$. The waveform ascended slowly, and the pulse pressure was unusually narrow. Phenylephrine $(0.1 \mathrm{mg})$ was given intravenously 3 times, and her systolic pressure increased by $20 \mathrm{~mm} \mathrm{Hg}$ after each dose; however, the abnormal wave form did not improve. The arterial line circuit was intact and exhibited no bending, kinking, or air bubbles. We conducted a flash test, which was normal. Because we initially suspected stenosis of the left radial artery, we inserted a second arterial catheter in her right radial artery; however, the pressure tracing revealed a similarly abnormal waveform (Fig. 1). The patient's bilateral ulnar pulses were palpated; however, her radial pulses were stronger and more easily felt. We suspected that the patient may have had a perfusion deficiency in the bilateral upper extremities; consequently, POCUS was immediately performed.

Using POCUS, we identified hypoplasia and stenosis of bilateral subclavian arteries and complete obstruction of bilateral common carotid arteries (Fig. 2). At around $3 \mathrm{~mm}$ in diameter, the bilateral subclavian arteries were around a quarter of the size of the accompanying subclavian veins. The diagnosis of bilateral common carotid artery obstruction was easily confirmed by color doppler imaging. Furthermore, bilateral vertebral arteries were significantly dilated with a diameter of approximately $7 \mathrm{~mm}$. Because both subclavian arteries were observed to be abnormally narrow via imaging, we believed that the blood pressure measurement in the bilateral upper extremities was not reliable. Therefore, we inserted a third arterial catheter in the patient's left dorsalis pedis artery. When the arterial blood pressure measured from her left radial artery was 
$81 / 56$ (mean 66) $\mathrm{mm} \mathrm{Hg}$, it was 66/47 (mean 55) $\mathrm{mm} \mathrm{Hg}$ from her right radial artery and 160/69 (mean 96) $\mathrm{mm} \mathrm{Hg}$ from her left dorsalis pedis artery (Fig. 3). Based on the POCUS findings, we determined that the blood pressure measured from her dorsalis pedis artery was most accurate for perioperative circulatory management. The patient emerged from anesthesia immediately after the operation was concluded, and no neurological deficits were observed.

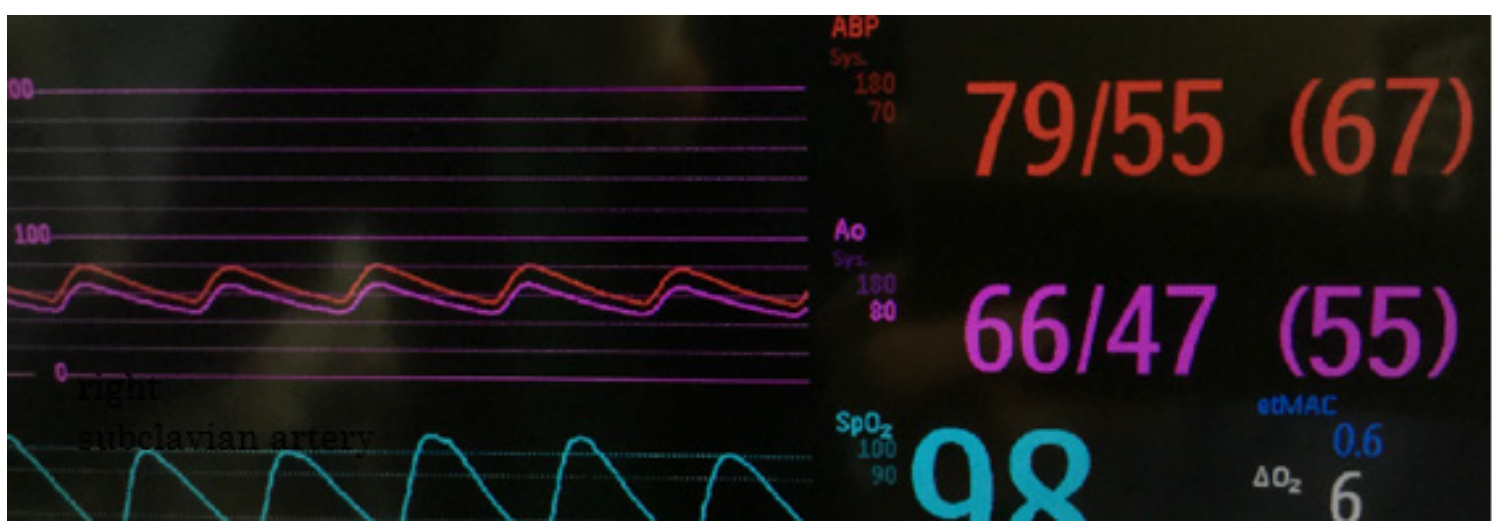

\begin{tabular}{l|}
\hline left \\
radial artery
\end{tabular}

\begin{tabular}{|l|}
\hline right \\
radial artery \\
\hline
\end{tabular}

Figure 1. Image shows similarly abnormal waveform of arterial blood pressure from bilateral radial arteries.
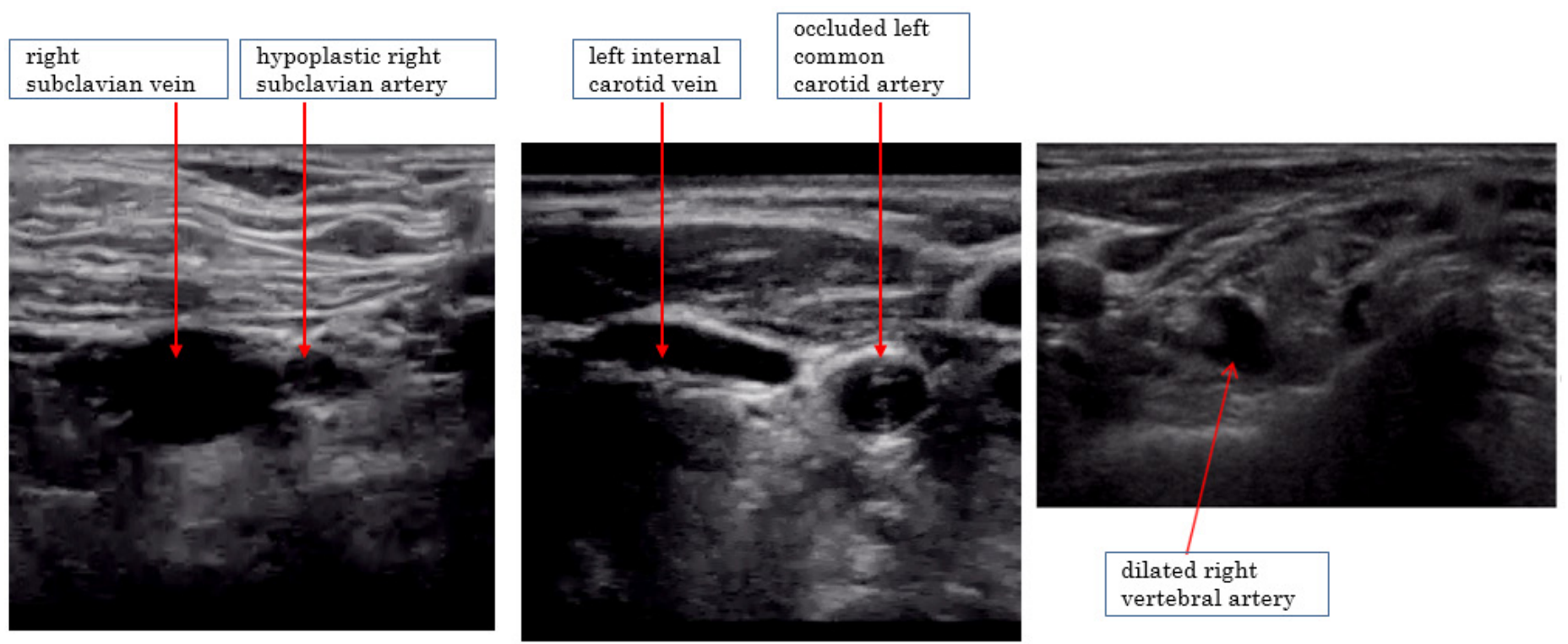

Figure 2. Ultrasonography of the neck. Hypoplasia and stenosis of bilateral subclavian arteries, complete obstruction of bilateral common carotid arteries, and dilated bilateral vertebral arteries were observed.

Due to the POCUS findings, we conducted a detailed examination by enhanced computed tomography (CT) postoperatively. All 3 branch vessels of her aortic arch (brachiocephalic trunk, left common carotid artery, and left subclavian artery) were completely occluded at their regions of origin, and well-developed collateral pathways supplied blood to her arms and brain (Fig. 4). A history of aortic arch syndrome (Takayasu's arteritis) was suspected.

\section{Discussion}

POCUS is being utilized more often in clinical settings [1-3]. In this case, bilateral subclavian artery stenosis was suspected based on abnormal pressure waveforms of bilateral radial arteries. POCUS was immediately performed, which revealed a previously undiagnosed vascular abnormality that could affect the patient's brain and upper extremities. Postoperative enhanced CT confirmed the findings identified by POCUS.

In POCUS findings, we could easily identify vascular abnormalities. The vessel walls of bilateral common carotid arteries were thickened circumferentially, and color doppler imaging showed complete occlusion. The bilateral subclavian arteries were only about $3 \mathrm{~mm}$ in diameter, and were clearly smaller than accompanying subclavian veins. Bilateral vertebral arteries, which are normally about $3 \mathrm{~mm}$ in diameter [4,5], were significantly dilated to approximately 7 $\mathrm{mm}$ in diameter.

In order to accurately interpret the POCUS findings, sufficient experience is required [1]. The common carotid and subclavian arteries are routinely identified by anesthesiologists during ultrasound- 
guided central venous catheter insertion, and the vertebral artery is frequently identified during ultrasound-guided brachial plexus blocks. Moreover, anesthesiologists often evaluate stenosis of the common carotid artery as part of perioperative POCUS. Because we routinely use ultrasonography and are accustomed to POCUS, we immediately noticed the abnormality of the arteries, which contributed to our ability to make this diagnosis.

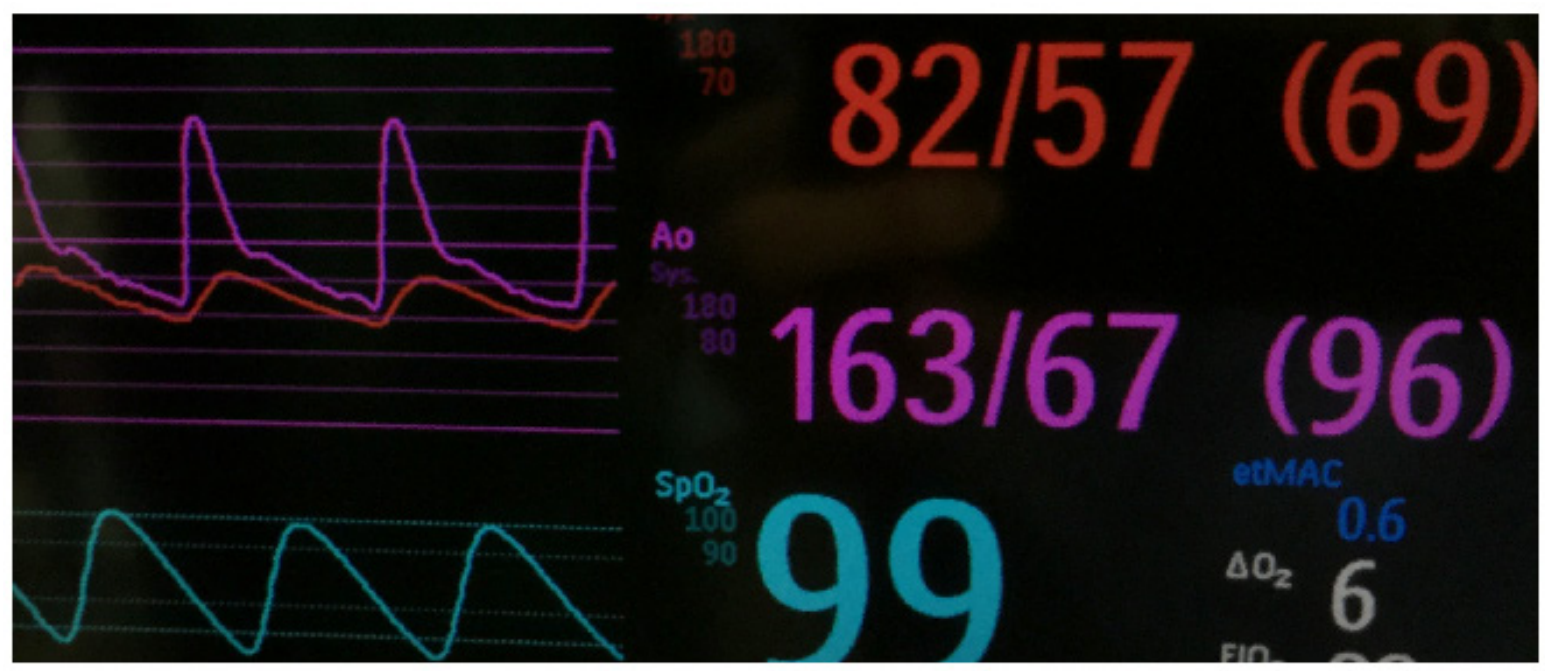

left

radial artery

left dorsalis

pedis artery

Figure 3. Image shows arterial blood pressure measured from the left radial artery and left dorsalis pedis artery.
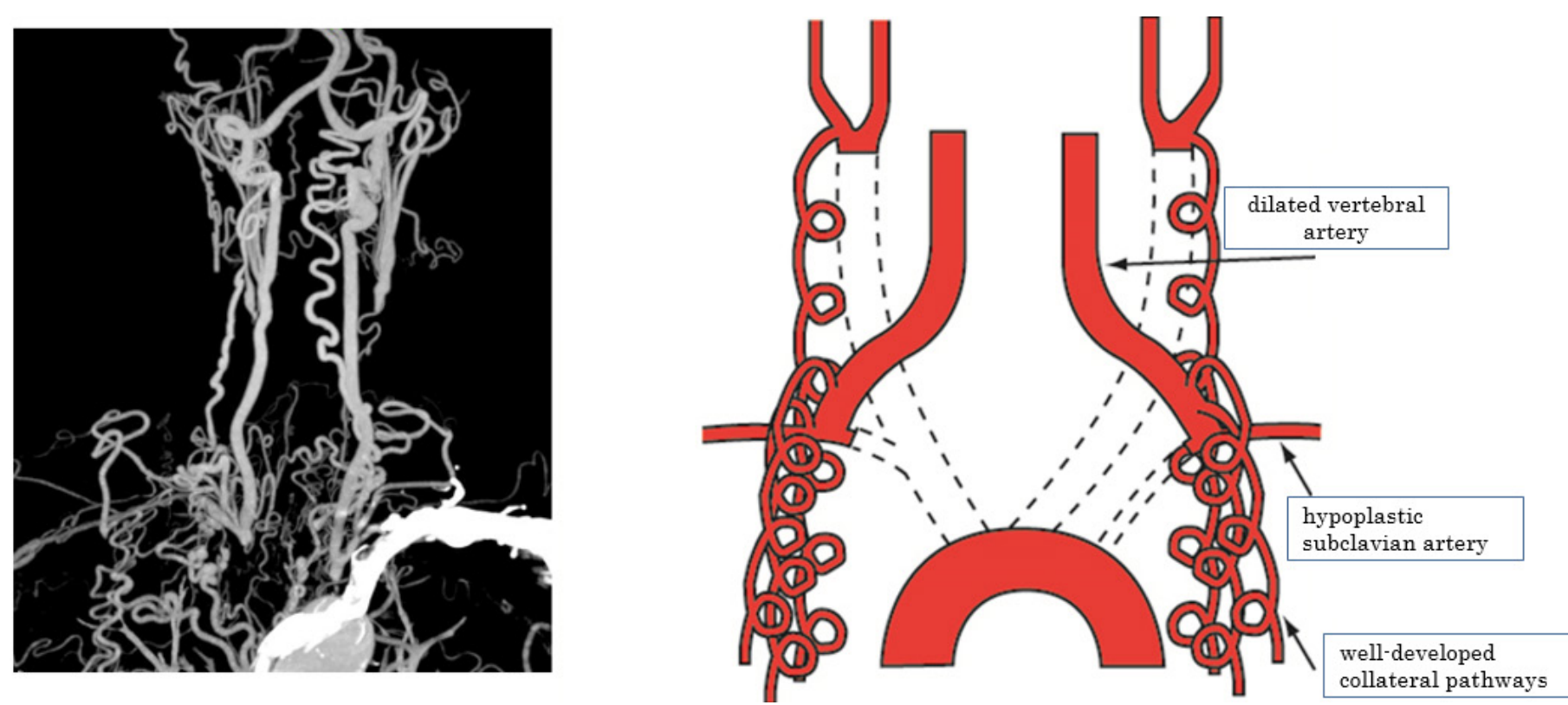

Figure 4. Computed tomography (CT) and schema. All three branch vessels of the aortic arch were totally occluded and well-developed collateral pathways supplied blood flow to her arms and brain.

Based on the POCUS findings, we relied on the lower extremity blood pressure for perioperative circulatory management. Because POCUS and preoperative head MRI results showed obstruction of the bilateral common carotid arteries and dilation of the bilateral vertebral arteries, the patient's cerebral blood flow was thought to depend on the vertebral arteries. We judged that only the upper extremities were hypotensive, and that the blood pressure measured from the lower extremities more accurately reflected the perfusion pressure of the patient's major organs. We investigated the regional oxygen saturation (rSO2) as an index of cerebral blood flow. However, it would have been difficult to monitor the patient's $\mathrm{rSO} 2$ in this case due to the location of the surgical field.

This case highlights how circulatory management that blindly gives priority to radial artery pressure could lead to end-organ hypertension, which could increase bleeding from the surgical field. In this case, the radial artery blood pressure was significantly lower 
than the blood pressure of the lower extremities (about 30-40 $\mathrm{mm} \mathrm{Hg}$ less in mean arterial pressure). We thought that tight blood pressure control was warranted, because excessive hypotension could have caused ischemia of the upper extremities, and hypertension could have induced surgical bleeding. Thus, we sought to maintain a mean arterial pressure in the lower extremities of approximately $80 \mathrm{~mm}$ $\mathrm{Hg}$ (130 mm Hg in systolic blood pressure), because at this pressure, the mean arterial pressure of the right radial artery stayed around 50 $\mathrm{mm} \mathrm{Hg}$. Although setting an appropriate target blood pressure was extremely difficult in the limited environment and information during surgery, we deemed this target blood pressure as appropriate because the patient had no definite history of hypertension and it would enable us to avoid excessive hypertension, which is important during craniotomies.

The patient was similarly managed in the intensive care unit postoperatively, and she experienced no bleeding, neurological problems, or other complications including major organ failure. A CT scan performed 6 days after the operation showed complete occlusion of her brachiocephalic trunk, left common carotid artery, and left subclavian artery at their regions of origin from the aortic arch. Numerous collateral pathways from arteries of the superior mediastinum and chest wall, which branched from the aorta, supplied blood to the aortic arch branch vessels. Collateral pathways also supplied blood to bilateral vertebral arteries.

Although there is no conclusive evidence that the patient's lower extremity blood pressure reflected her cerebral blood pressure and that our target arterial pressure was exactly appropriate, our careful circulatory management that avoided excessive hypertension and hypotension during the perioperative period, might have contributed to the patient's good postoperative neurological outcome. Because preoperative head MRI data revealed abnormalities in the bilateral common carotid arteries and bilateral vertebral arteries, further detailed evaluation of other blood vessels below the neck by enhanced CT should have been considered before operation and may be important for similar situations in the future.

After consulting with a cardiothoracic surgeon, the patient subsequently confirmed that she had a weak pulse during a medical exam when she was in her twenties. Since this issue had been identified in the patient's youth and because she had few risk factors of arteriosclerosis such as hypertension, diabetes mellitus, and smoking, a history of aortic arch syndrome (Takayasu's arteritis) was suspected. There were, however, few findings and symptoms suggesting active arteritis such as an elevation of inflammatory markers, and the patient denied further evaluation and treatment; therefore, it is unknown whether the complete occlusion of the aortic arch branch vessels was caused by aortic arch syndrome.

Some reports have described anesthetic management of patients diagnosed with stenosis and/or obstruction of the aortic arch branch vessels that occurs with aortic arch syndrome (Takayasu's arteritis) $[6,7]$. These reports highlight the usefulness of ultrasonography to determine the best site for blood pressure monitoring [6] and the efficacy of rSO2 monitoring as an index of cerebral blood flow [7]. However, to the best of our knowledge, there are few reports in which a previously undiagnosed stenosis and/or obstruction of the aortic arch branch vessels was identified by POCUS during a perioperative period. Because POCUS has the potential to significantly improve perioperative care, anesthesiologists should strive to acquire this skill. We expect future clinical studies and cases that involve POCUS during surgery will further demonstrate how its use can improve perioperative outcomes.

\section{Acknowledgements}

We would like to thank our patient for providing to publish this case report. We would also like to thank Editage (www.editage.jp) for English language editing.

\section{Funding}

The authors declare that they have no funding.

\section{Authors' contributions}

MI and RY wrote the draft of the manuscript. TY made the figures. IW reviewed the manuscript. The final version of the manuscript was approved by all authors.

\section{Ethics approval and consent to participate}

Not applicable.

\section{Consent for publication}

Written informed consent was obtained from the patients for publication of this case report and accompanying images.

\section{Competing interests}

The authors declare that they have no competing interests.

\section{Author details}

Department of Anesthesia, Yokohama Minami Kyosai Hospital, 1-21-1 Mutsuurahigashi, Kanazawa-ku, Yokohama 2360037, Japan

\section{Abbreviations \\ POCUS: Point-of-care ultrasound \\ MRI: magnetic resonance imaging \\ ICU: intensive care unit \\ CT: Computed tomography \\ rSO2: regional oxygen saturation}

\section{References}

1. Moore CL, Copel JA. (2011) Point-of-Care Ultrasonography. N Engl J Med. 364: 749-757. [Crossref]

2. Johnson DW, Oren-Grinberg A. (2011) Perioperative point-of-care ultrasonography: the past and the future are in anesthesiologists' hands. Anesthesiology. 115(3): 4602. [Crossref]

3. Holm JH, Frederiksen CA, Juhl-Olsen P, Sloth E. (2012) Perioperative Use of Focus Assessed Transthoracic Echocardiography (FATE). Anesth Analg. 115(5): 1029-32. [Crossref]

4. Park JH, Kim JM, Roh JK. (2007) Hypoplastic vertebral artery: frequency and associations with ischaemic stroke territory. J Neurol Neurosurg Psychiatry. 78(9): 954-8. [Crossref]

5. Ogeng'o J, Olabu B, Sinkeet R, Ogeng'o NM, Elbusaid H. (2014) Vertebral Artery Hypoplasia in a Black Kenyan Population. Int Sch Res Notices. 2014: 934510. 
Masafumi Idei (2019) Complete Occlusion of Aortic Arch Branch Vessels Diagnosed by Intraoperative Point-of-Care Ultrasonography: A Case Report

6. Narasimha PK, Chaudhuri S, Joseph TT (2013) Utility of intra-operative ultrasound in choosing the appropriate site for blood pressure monitoring in Takayasu's arteritis. Indian J Anaesth. 57(1): 66-8. [Crossref]
7. Xiao W, Wang T, Fu W, Wang F, Zhao L. (2016) Regional cerebral oxygen saturation guided cerebral protection in a parturient with Takayasu's arteritis undergoing cesarean section: a case report. J Clin Anesth. 33: 168-72. [Crossref]

\section{Citation:}

Masafumi Idei, Rie Yoshida, Tasuku Yoshida, Itaru Watanabe (2019) Complete Occlusion of Aortic Arch Branch Vessels Diagnosed by Intraoperative Point-of-Care Ultrasonography: A Case Report. J Clin Res Med Volume 2(1): 1-5. 